\title{
Response to 'Comment on "Adjuvant Trastuzumab Therapy for Early HER2-Positive Breast Cancer in Iran: A Cost- Effectiveness and Scenario Analysis for an Optimal Treatment Strategy"'
}

\author{
Amir Ansaripour ${ }^{1}$ (D) Carin A. Uyl-de Groot ${ }^{1}$ - W. Ken Redekop ${ }^{1}$
}

(C) Springer International Publishing AG, part of Springer Nature 2018

\section{Key Points}

It is necessary to include future non-breast cancer costs in cost-effectiveness analyses.

Inclusion of future non-breast cancer costs does not change our conclusions about the optimal treatment strategy in Iran.

\section{Dear Editor,}

We welcome the comments on our article "Adjuvant trastuzumab therapy for early HER2-positive breast cancer in Iran: a cost-effectiveness and scenario analysis for an optimal treatment strategy" [1] by Gershon and Berchenko [2], who express the importance of including future nonbreast cancer costs in our cost-effectiveness analysis when downstream costs ${ }^{1}$ are included in the analysis. As such, they conclude that the optimal treatment regimen would be 1 year of trastuzumab use in the early stage with "only a partial treatment in progressed stages". In this letter, we respond to their commentary and discuss how much their ideas can impact our results and conclusions.

This reply refers to the article available at https://doi.org/10.1007/ s40273-018-0621-x.

Amir Ansaripour

ansaripour@eshpm.eur.nl

1 Institute for Medical Technology Assessment, Erasmus

School of Health Policy and Management, Erasmus

University Rotterdam, PO Box 1738, 3000 DR Rotterdam,

The Netherlands
In general, we agree with the opinion by Gershon and Berchenko regarding the necessity of including future nonbreast cancer costs in cost-effectiveness analyses. Some policy makers also believe that they should be included in these analyses and have therefore included them in national guidelines [3]. To explore the impact of future non-breast cancer costs on the findings of our study, we first checked if age-specific, future non-breast cancer costs are available in Iran but, as far as we know, there is no reliable source for these data. Therefore, we assumed that annual future nonbreast cancer costs are equal to the annual total health expenditure per capita, which, when based on the percentage of the Iranian gross domestic product per capita spent on healthcare multiplied by the gross domestic product, is $€ 497$ (7.1\% of $€ 7000$ ) [4]. We then updated the results of our study using this value.

Figure 1 provides the results of the updated cost-effectiveness analysis as well as our previous results [1]. As Fig. 1 shows, the inclusion of future non-breast cancer costs has very little effect on the results, although it does lead to a slight reduction in the negative correlation between incremental costs and incremental effectiveness (i.e., the 'clouds' in Fig. 1b are not as slanted to the right as those in Fig. 1a).

While some might expect that the relatively low costs per year of $€ 497$ limit the effect of adding future non-breast cancer costs on the results, this is just one of the factors that play a role. Table 3 in our paper [1] shows that the lifeyears gained from using trastuzumab is clinically important but not large. For example, the base-case analysis results in total life-years following 1 year of trastuzumab vs. no trastuzumab of 15.82 vs. 14.41 , which means a gain in life-

\footnotetext{
${ }^{1}$ Here, we use the term "downstream costs" instead of "terminal costs" used by Gershon and Berchenko in their commentary.
} 


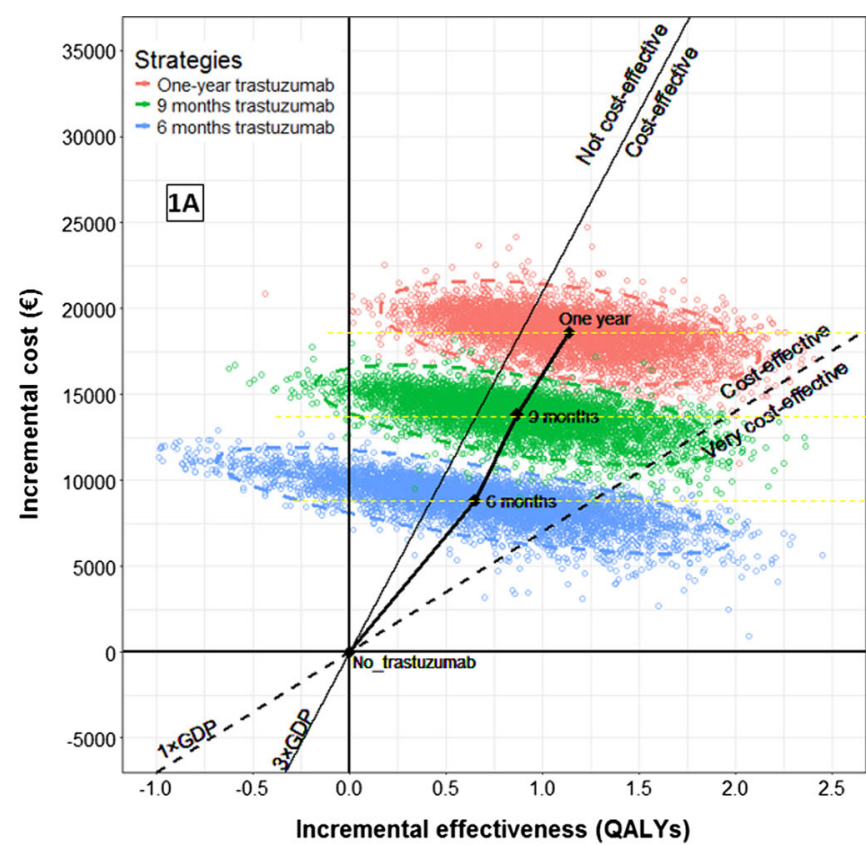

Fig. 1 Cost-effectiveness planes showing the impact of including non-breast cancer costs on the cost effectiveness of trastuzumab in Iran; a shows the results when non-breast cancer costs are excluded

years of 1.41. While women who received 1 year of trastuzumab will incur extra costs because of a longer life, this will amount to $€ 701$ (1.41 year $* € 497$ per year); this amount becomes smaller if we include discounting. Last, it is worth pointing out that these costs are relatively small compared with the cost of trastuzumab $(€ 22,992)$, which is incurred in the first year. This is the primary reason why their inclusion has little impact on the cost-effectiveness results.

Another point we would like to address is the suggestion by Gershon and Berchenko that policy makers should consider partial treatment of patients with advanced breast cancer. While the idea of partial treatment might be mathematically relevant, greater clarification is needed about its definition and how it could be implemented in routine practice. Possible interpretations of the term of "partial treatment" include less chemotherapy (doses or frequency) per patient and treatment of only some patients (e.g., using the principles of triage and stratified medicine). Either way, clinicians would have to be willing to develop a method for defining the term and designing a protocol (preferably based on the literature and daily practice) to implement this successfully.

In conclusion, we agree with the authors that it is necessary to include future non-breast cancer costs in costeffectiveness analyses. However, their inclusion will not always affect the results or the conclusions about the cost

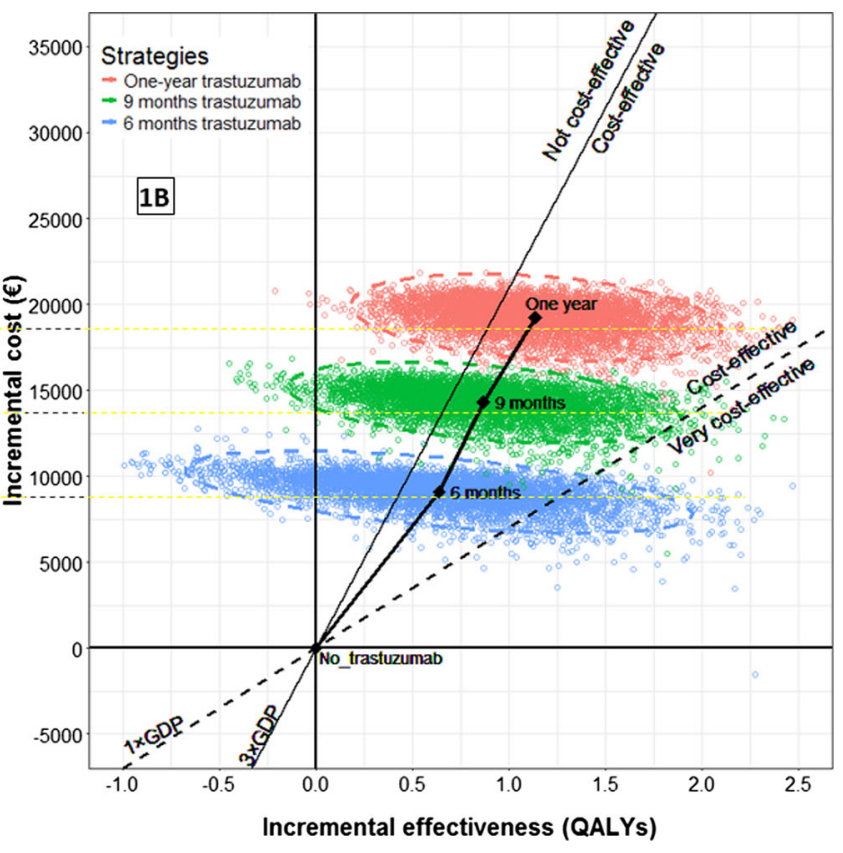

[1] while b shows the results when they are included. GDP gross domestic product, $Q A L Y s$ quality-adjusted life-years

effectiveness of one treatment strategy vs. another. The magnitude of the impact of including the costs is determined by different factors, and their inclusion does not change our conclusions about the optimal treatment strategy of trastuzumab in Iran.

\section{Compliance with Ethical Standards}

Funding No sources of funding were received for the preparation of this response.

Conflict of interest Amir Ansaripour, Carin A. Uyl-de Groot, and W. Ken Redekop have no conflicts of interest directly relevant to the content of this response.

\section{References}

1. Ansaripour A, Uyl-de Groot C, Redekop WK. Adjuvant trastuzumab therapy for early HER2-positive breast cancer in Iran: a costeffectiveness and scenario analysis for an optimal treatment strategy. Pharmacoeconomics. 2018;36:91-103.

2. Gershon N, Berchenko Y. Optimality, future costs and terminal costs. Pharmacoeconomics. 2018.

3. Versteegh M, Knies S, Brouwer W. From good to better: new Dutch guidelines for economic evaluations in healthcare. Pharmacoeconomics. 2016;34:1071-4.

4. The World Bank. In: GDP per capita (current US\$). 2015. http:// data.worldbank.org/indicator/NY.GDP.PCAP.CD. Accessed 20 Oct 2015. 